\title{
Stillen: alles klar - oder doch nicht?
}

\author{
Das Stillen von Kindern ist ein unspektakuläres Thema. Die Meinung ist ver- \\ breitet, Stillen funktioniere problemlos, da es doch «natürlich» sei. Die Auto- \\ rin zeigt, dass dem nicht immer so ist und plädiert für mehr Unterstützung \\ durch Beratung und einen besseren Schutz des Stillens durch rechtliche Mass- \\ nahmen, auch in Ländern wie der Schweiz.
}

Rebecca Norton, Fachperson Ernährung bei Terre des hommes

\footnotetext{
Ausschliesslich stillen: nur Muttermilch ohne Zugabe von Wasser, Saft, Tee oder anderen Getränken; nur medikamentöse Tropfen sind erlaubt.
}

Korrespondenz:

Terre des hommes

Köchlistrasse 2

Postfach

CH-8021 Zürich

www.tdh.ch
Stillen ist ein breitgefächertes Thema: Es ist ein Thema der Medizin, wenn es um die Gesundheit des Säuglings geht. Es ist ein Thema des Rechts, wo es um das Recht jedes Kindes, gestillt zu werden, geht. Es ist ein Thema der Politik, wo es um Mutterschaftsurlaub geht, und es ist sogar ein Thema der Globalisierung, wenn es um die Macht von Konzernen, die weltweit Milchpulver verkaufen, geht. 10,8 Millionen Kinder weltweit sterben jedes Jahr vor ihrem fünften Geburtstag. Die Hälfte davon stirbt an Infektionskrankheiten, d.h. an den Folgen von Mangelernährung, die das Immunsystem der Kleinen schwächt. Paradoxerweise könnte die Mehrheit dieser Todesfälle durch relativ einfache Massnahmen verhindert werden. Gemäss Angaben der Fachzeitschrift «The Lancet» (Child Survival Series 2003) könnten 1,3 Millionen Kinder überleben, wenn sie richtig gestillt würden [1]. Dies sind mehr als zehn Prozent aller Todesfälle. Die WHO empfiehlt deshalb das ausschliessliche* Stillen während der ersten sechs Monate und danach die Einführung von angemessener Beikost bei fortgesetztem Stillen bis zwei Jahre oder mehr.

Die wichtigsten Vorteile des Stillens für Mutter und Kind seien an dieser Stelle nochmals kurz in Erinnerung gerufen:

1. Für die Gesundheit und die Entwicklung des Kindes: weniger Durchfall, Atemwegerkrankungen, Ohrenentzündungen und weitere übertragbare Infektionen. Das Kind entwickelt sich intellektuell und motorisch besser; ausserdem erkrankt es weniger häufig an chronischen Krankheiten und nicht übertragbaren Infektionen (Allergien, Fettleibigkeit, Diabetes, Gefässerkrankungen und Krebs).

2. Für die Mutter: tieferes Risiko von Anämie und Blutungen nach der Geburt sowie von Brust- und Eierstockkrebs. Ausserdem erfol-

\section{L'allaitement maternel - encore du pain sur la planche!}

L'allaitement maternel $n$ 'est pas une thématique très en vogue. «Allaiter est un acte naturel, ça marche tout seul» - est une opinion populaire. Pourtant, selon l'auteur, cela n'est pas toujours le cas. Bien plus d'efforts pourraient être faits pour promouvoir l'allaitement maternel, surtout pour généraliser le soutien pratique aux femmes allaitantes par des personnes formées en conseil en lactation, et protéger l'allaitement maternel via la mise en œuvre de mesures législatives spécifiques comme le congé maternité et le Code International de commercialisation des substituts du lait maternel et résolutions pertinentes de I'Assemblée générale de l'Organisation mondiale de la santé, plus souvent appelé «le Code». Ceci est valable dans tous les pays, en Suisse aussi. Les professionnels de santé en particulier des médecins jouent un rôle clef, car ils ont le pouvoir d'informer les femmes, notamment de les référer en cas de besoin, aux conseillères en lactation ou sages-femmes qui pourront leur offrir un conseil pratique de qualité. La Fondation Terre des hommes met un accent particulièrement important sur la promotion, le soutien et la protection de l'allaitement maternel dans ses projets de santé. 


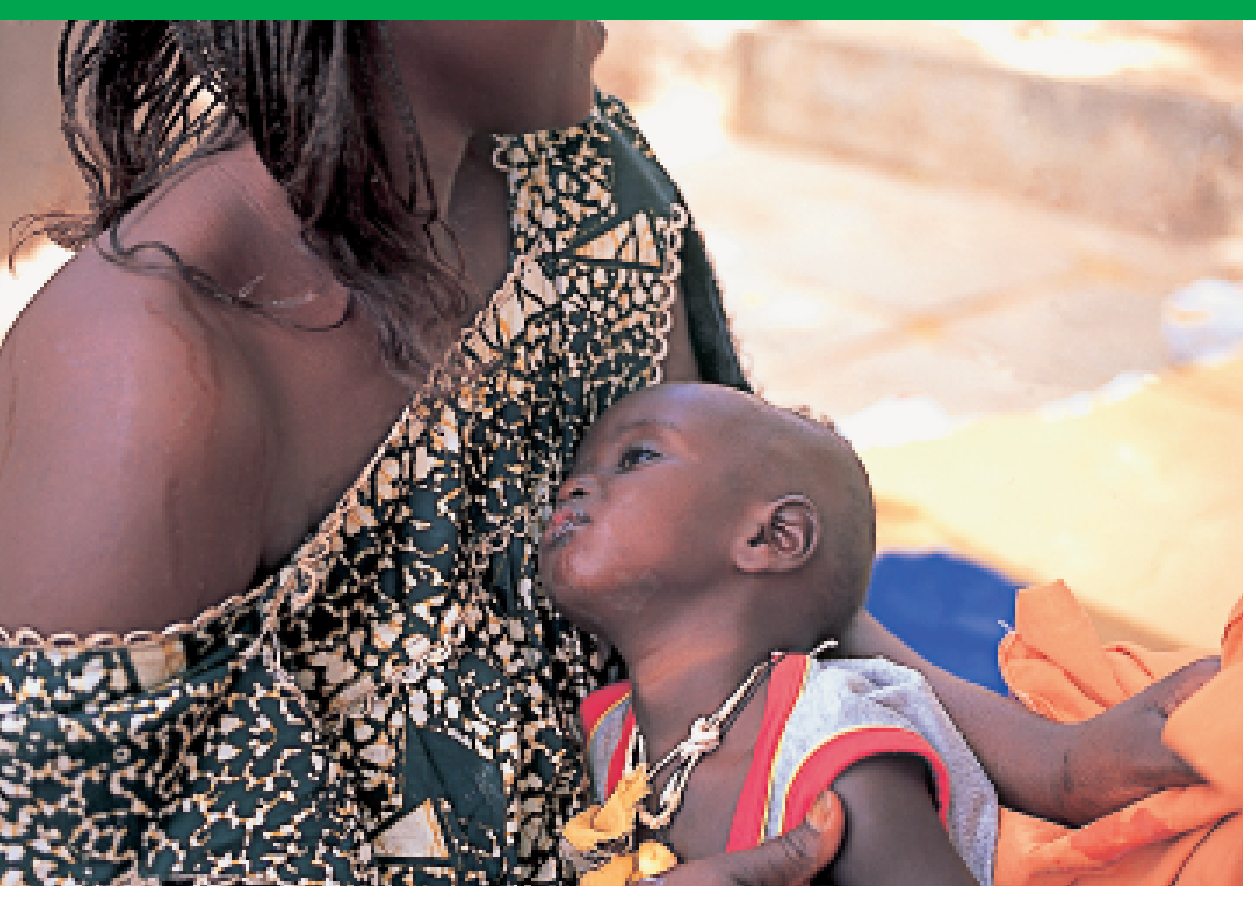

Gemäss WHO könnten jährlich 1,3 Millionen Todesfälle bei Kindern unter fünf Jahren durch korrektes Stillen verhindert werden.

Foto: Mikel Arrazola

Die 10 wichtigsten Punkte des Kodexes:

1. Keine Werbung in der Öffentlichkeit.

2. Keine Gratismuster an Mütter.

3. Keine Werbung innerhalb des Gesundheitswesens.

4. Kein Kontakt von Firmenpersonal zu Müttern.

5. Keine Geschenke und Muster an Gesundheitspersonal.

6. Keine Idealisierung in Text und Bild der künstlichen Nahrung auf den Verpackungen.

7. Informationen an das Gesundheitspersonal müssen sich auf wissenschaftliche Befunde und Tatsachen beschränken.

8. Informationsmaterial und Etiketten müssen auf die Vorteile und Überlegenheit der Muttermilch hinweisen und vor den Gesundheitsrisiken und Kosten des Ersatzproduktes warnen.

9. Keine Werbung für ungeeignete Produkte wie gesüsste Kondensmilch für Säuglinge.

10. Alle Produkte müssen von guter Qualität sein. Die klimatischen sowie Lagerungsbedingungen im Zielland müssen berücksichtigt werden.

Internationaler Kodex und Resolutionen zur Vermarktung von Muttermilchersatzprodukten: www.who.int und_www.ibfan.org.

gen die Schwangerschaften in grösseren $\mathrm{Ab}$ ständen.

3. Ökonomische und ökologische Vorteile: Weniger Gesundheitskosten; weniger Ausgaben für Säuglingsmilchpulver, Utensilien und Energie für deren Zubereitung; weniger Abfall.

Jedes Kind hat das Recht «auf das erreichbare Höchstmass an Gesundheit», stipuliert Artikel 24 der UNO-Konvention über die Rechte des Kindes. Die Förderung, Unterstützung und der Schutz des Stillens ist eine der wichtigsten Massnahmen für die Gewährleistung dieses Rechts.
Terre des hommes - Kinderhilfe schenkt deshalb dem Stillen in den Gesundheitsprojekten für Mutter und Kind höchste Aufmerksamkeit.

\section{Internationale Bemühungen verstärken}

Die neue Strategie der WHO zur Säuglings- und Kleinkindernährung (2003) will die Anstrengungen auf dem Gebiet des Stillens fördern. Zwar hat sich schon einiges getan, doch fehlt es an der Durchsetzung des Internationalen Kodexes (siehe Kasten), an einem ausreichenden Mutterschaftsschutz und an der ganz konkreten Beratung von Müttern, die Schwierigkeiten mit dem Stillen haben. Wenn zum Beispiel eine junge Mutter verunsichert ist, weil ihre Milch auch am dritten Tag noch nicht richtig fliesst oder weil sie nicht weiss, dass es normal ist, dass ihr Kind in den ersten Tagen ein wenig an Gewicht verliert, wird sie vielleicht zu schnell zu Milchpulver greifen.

In den meisten Projektländern von Terre des hommes stillt jede Mutter, doch ist die Rate derjenigen, die ausschliesslich stillen und keine Beikost verwenden, relativ gering (Burkina Faso 6\%, Senegal 24\%, Mauretanien 20\%). Weltweit erhalten weniger als $35 \%$ der Säuglinge in den ersten vier Monaten ausschliesslich Muttermilch. Beikost wird häufig zu früh verwendet, und viele Mütter stillen zu früh völlig ab. Auch in der Schweiz steht es damit nicht zum besten: Im Jahre 2003 wurden ganze 24\% der Säuglinge bis zum sechsten Monat ausschliesslich gestillt, und weniger als $62 \%$ der Mütter stillten länger als sechs Monate [2].

Unter den vielen Faktoren, die das Stillen beeinflussen, spielt das Gesundheitspersonal eine wichtige Rolle. Doch Ärzten und Pflegepersonal fehlt häufig eine praktische Ausbildung in diesem Bereich. Auch nimmt die Beratung und das Zuhören viel Zeit in Anspruch. In der Schweiz können zwar Mütter - dank dem gut entwickelten Netz von Stillberaterinnen und dank gut ausgebildeten Hebammen - Probleme beim Stillen meistern. Allerdings bleiben immer noch $\mathrm{zu}$ viele mit ihren Schwierigkeiten allein. Daher ist die Zusammenarbeit von Stillberaterinnen, Hebammen und Gesundheitspersonal von grosser Bedeutung. Vor allem Ärzte können einen grossen Einfluss auf Mütter haben.

Terre des hommes bildet in ihren Projekten seit zwei Jahren Gesundheitspersonal in praktischer Stillberatung aus. Beratung bedeutet dabei aktives Zuhören, Schaffen eines Vertrauensverhältnisses, Loben von richtigem Verhalten und praktische Hilfe bei allen Problemen rund ums Stillen. Die meisten Probleme können ganz einfach mit dem richtigen Anlegen des Kindes an die Brust gelöst werden. 
Die WHO und Unicef haben Instrumente zur Ausbildung von Stillberaterinnen erstellt. Terre des hommes fördert nicht nur das Stillen in den eigenen Ernährungsprojekten (Afghanistan, Guinea, Senegal, Burkina Faso, Benin), sondern hält auch die Spitäler, mit denen sie zusammen arbeitet, dazu an, das Label «Stillfreundliche Geburtsklinik» für Stillberatung von Unicef und WHO zu erwerben.

\section{Häufig vernachlässigt: der Schutz des Stillens}

Die kommerzielle Werbung für Milchpulver und andere Nahrungsmittel für Säuglinge übt einen erheblichen Einfluss auf die Haltung der Mütter zum Stillen aus. Um das Stillen vor den aggressiven Praktiken der Ersatznahrungshersteller zu schützen, hat die Vollversammlung der WHO im Jahr 1981 den Internationalen Kodex zur Vermarktung von Muttermilchersatzprodukten verabschiedet. Alle zwei Jahre werden Resolutionen hinzugefügt.

Der Kodex ist eine Empfehlung zur Volksgesundheit und richtet sich an Regierungen, an Gesundheitspersonal und an Konsumenten, ebenso an Hersteller und Verteiler von Ersatzprodukten für Muttermilch.

Die Empfehlungen lauten:

- für Regierungen: den Kodex in die Gesetzgebung integrieren und seine Respektierung überwachen;

- für Hersteller und Verteiler: den Kodex beachten;

- für das Gesundheitspersonal: den Kodex beachten und seine Anwendung überprüfen.

Bereits 32 Länder haben alle Artikel des Kodexes in ihre Gesetzgebung integriert und überwachen

\section{Stillberatung im Spital}

In Guinea (Westafrika) führt Terre des hommes Kurse in praktischer Stillberatung durch. Im Spital der Provinzstadt Donka konnten die Teilnehmer/innen des Stillberatungskurses miterleben, wie wichtig ihre Tätigkeit für eine junge Mutter sein kann. Ihr Kind war vor mehr als 24 Stunden durch einen Kaiserschnitt auf die Welt gekommen und hatte noch nicht getrunken. Bisher hatte sich niemand die Zeit genommen, der Mutter mit dem Stillen zu helfen. Unter der Aufsicht der Kursleiterin hörte sich ein Teilnehmer zuerst die Schwierigkeiten der Mutter an. Sie konnte wegen der Schmerzen kaum sitzen. Der Teilnehmer empfahl ihr, sich für das Stillen hinzulegen und zeigte ihr, wie sie das Kind an die Brust legen sollte. Dadurch hatte die Mutter keine Schmerzen und das Kind lag in der richtigen Position. Der Teilnehmer ermutigte sie, das Stillen nicht aufzugeben und lobte sie für ihren Durchhaltewillen. Durch diese Unterstützung fand die Mutter selbst die ideale Stillposition, und nach kurzer Zeit sog das Baby zufrieden an der Brust. seine Anwendung. Brasilien, Indien und Sri Lanka haben ihre Gesetze kürzlich sogar verschärft. In der Schweiz ist der Kodex noch nicht Bestandteil der Gesetzgebung. Es existiert ein «Verhaltenskodex der Hersteller bezüglich der Vermarktung von Säuglingsnahrung» (1994 revidiert), doch er hat keinen gesetzlichen Charakter.

Die Erfahrung hat gezeigt, dass die Übernahme des Kodexes ins nationale Gesetz und dessen Überwachung die Babynahrungsmittelhersteller zur moderaten Anwendung ihrer Werbepraktiken veranlasst. In Ägypten hat Terre des hommes - in Zusammenarbeit mit IBFAN (International Baby Food Action Network) und dem Gesundheitsministerium - an einer Untersuchung zur Einhaltung des Kodexes teilgenommen. IBFAN erstellt weltweit regelmässig Rapporte über die Verstösse der Industrie gegen den Kodex.

Aber auch ein genügender Mutterschaftsschutz ist ausschlaggebend, um der berufstätigen Mutter das ausreichende Stillen zu ermöglichen. 2004 hat auch die Schweiz den Minimalstandard der internationalen Konvention zum Mutterschutz der ILO (Internationale Arbeitsorganisation), nämlich 14 Wochen Mutterschaftsurlaub, ins Gesetz übernommen. Ein Fortschritt, obwohl Mütter in vielen Ländern einen noch viel weitergehenden Schutz geniessen.

Der Schutz, die Förderung und Unterstützung des Stillens sind Schlüsselinterventionen, um die Säuglingssterblichkeit zu senken und die Gesundheit und Entwicklung des Babys zu fördern. Mütter brauchen konkrete Beratung und Unterstützung für erfolgreiches Stillen sowie Schutz durch rechtliche Schritte, die auch überwacht werden müssen.

\section{Literatur}

1 Black RE, Morris SS, Bryce J. Where and why are 10 million children dying every year? Lancet. 2003;361:2226-34; Jones G, Stekettee RW, Black RE, Bhutta ZA, Morris SS, the Bellagio Child Survival Study Group. How many child deaths can we prevent this year? Lancet. 2003;362:65-71; Bryce J, el Arifeen S, Pariyo G, Lanata CF, Gwatkin D, Habitch JP; the Multi-Country Evaluation of the IMCI Study Group. Reducing child mortality: can public health deliver? Lancet. 2003;362:159-64; Victora CG, Wagstaff A, Schellenberg JA, et al. Applying an equity lens to child health and mortality: more of the same is not enough. Lancet. 2003;362:233-41; The Bellagio Study Group on Child survival. Knowledge into action for child survival. Lancet. 2003;362:323-7.

2 Dratva J, Merten S. Säuglingsernährung in den ersten 9 Lebensmonaten. Basel: Institut für Sozialund Präventivmedizin; 2004. 